(RESEARCH ARTICLE)

\title{
Feasiblity study of psychological approach using robotics for education and communication
}

\author{
Sone Junji ${ }^{*}$, Katagami Daisuke, Ogai Yuta, Tomoto Takahito, Obo Takenori and Udagawa Yoshihisa \\ Faculty of Engineering Tokyo Polytechnic University, Atsugi, Kanagawa, Japan.
}

Publication history: Received on 01 January 2020; revised on 19 January 2020; accepted on 20 January 2020

Article DOI: https://doi.org/10.30574/gjeta.2020.2.1.0001

\begin{abstract}
The psychological approach to education and communication using robotics systems based on learning theory, humanagent interaction, biological analysis, and cognitive science is becoming increasingly important in activities such as education and driving assistance. We are developing these systems by integrating advanced topics of information, biological, and robotics research.
\end{abstract}

Keywords: Education; Communication; Robotics; Feasibility research

\section{Introduction}

Recently, many communication and intelligent robot researches have been proposed. Group behavior with cooperation was considered by CEBOT [1]. Asama et al. considered efficient negotiation which is realized by a newly developed communication functionality called groupcast and a learning mechanism utilizing historical records as ACTRESS [2]. Blackboard-like system is used to coordinate planning and scheduling the tasks by GOFER [3]. Steels considered cooperation between distributed agents through self-organization [4]. Kube and Zhang mentioned behaviour based robots to perform tasks without centralized control [5]. Dudek mentioned taxonomy for the characterization of multiagent robotic systems [6]. In cooperative robotics, agent modeling has been explored by Donald [7]. Reinforcement learning was considered by Barto et al. [8] and Parker [9]. Littman considered framework for multi-agent reinforcement learning [10]. Distributed Artificial Intelligence (DAI), Distributed Problem Solving (DPS) and Multiagent Systems (MAS) discussed by CAO [11]. Fujimoto developed multiple robot collaboration system using the database and task scheduler. A collaborative task experiment by multiple robots in a human environment using the Kukanchi System [12]. Asada mentioned motor representation and spatial perception is one of the most fundamental issues of Cognitive Developmental Robotics (CDR). But he did not proceed synthetic approaches because lack of good platforms [13]. These researches are basic element of human and robot communications and nowadays we have basic platform of robotics system. These must have the ability to interact more naturally with humans by using verbal, nonverbal representation and knowledge database. In this study, we aim to developed an education and communication system to improve students' motivation and their learning effects, integrating information technology, robotics and artificial intelligence. Moreover, we focus on the system design based on the effect of color that can affect students' psychological and behavioral responses $[14,15]$.

In this paper we present a brief introduction to the major technological elements in our research and provide some discussion on the color effect in an educational environment in terms of behavioral and biological information analysis, data mining, and human-robot interaction.

\footnotetext{
${ }^{*}$ Corresponding author

E-mail address: sone@cs.t-kougei.ac.jp
} 


\section{Research Elements}

We are researching five research themes for education and communication with human using robotics by using color psychological science.

\subsection{Influence of Robot Expressing Atmosphere Using Utterance Mind}

It has been clarified that a person unconsciously expresses the feeling "utterance mind" such as "I want to talk" when acting on speech as behavior. In this research, paying attention to the concept of "utterance mind", it is thought that a robot can read human atmosphere by implementing behavior that expresses "utterance mind" on the robot [16]. Figure 1 shows emotion recognition experiment system.

\subsection{Heart Rate Variability Analysis with Pneumatic Pressure Sensor}

We developed a measurement system with pneumatic pressure sensor for heart rate variability analysis (Fig.2). Heart rate variability (HRV) analysis is a non-invasive measure for assessing the autonomic nervous system (ANS) activity [17]. The pressure sensor can detect the ballistocardiograph (BCG) signal caused by bodily movement cyclically arising from the ejection of blood into the great vessels with each heartbeat. The measurement system can gather the data of the involuntary movements without any wearable device.

\subsection{Data Mining for Discovering Educational Rules from Log Data}

Through practices of the education and communication robots, large amounts of log data are generated. They are then used as inputs for mining or discovering educational rules from the log data of experiments. A text matching algorithm using the Longest Common Subsequence (LCS), a well-known algorithm of dynamic programming, is developed to provide partial matching functionality of texts, and then it is incorporated in an Apriori-based data mining algorithm to discover educational rules derived from experiments using the proposed robots for educational communication. At the first stage of development, the data mining algorithm is applied for finding programming patterns and rules using a package of Java programs composed of about 370,000 lines of source code, i.e., Java SWING graphics package. Figure 3 shows the example of co-occurrence words extraction and their graphic representation. Implementation of the following functions is in progress for the purpose:

- Co-occurrence words extraction and their graphic representation,

- Statistical analysis of co-occurring words and rule generation,

- Support function for combining rules to generate higher rules or knowledge on education and communication.

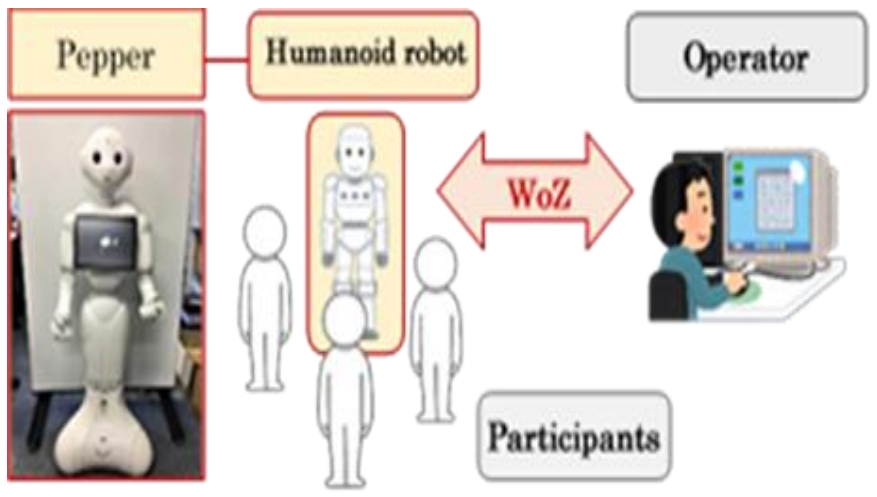

Figure 1 Emotion recognition experiment system. 


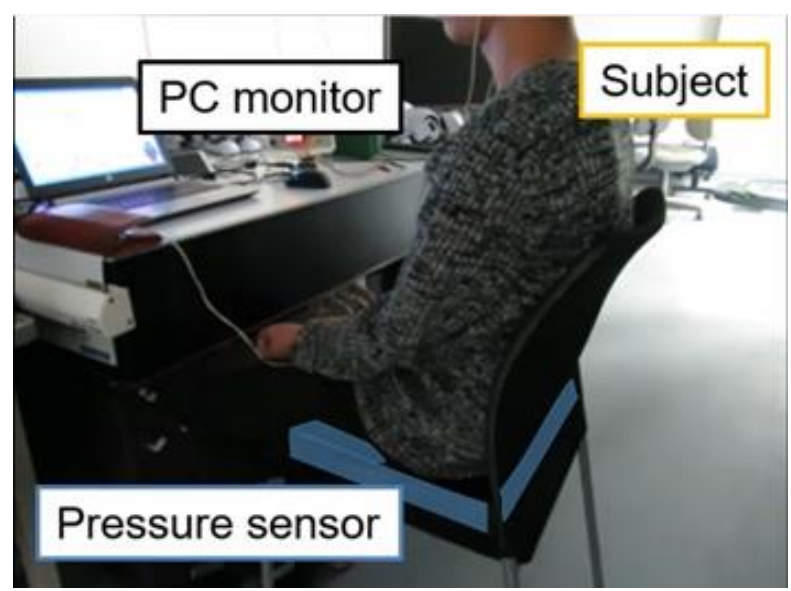

Figure 2 Measurement system with the pneumatic pressure sensor.

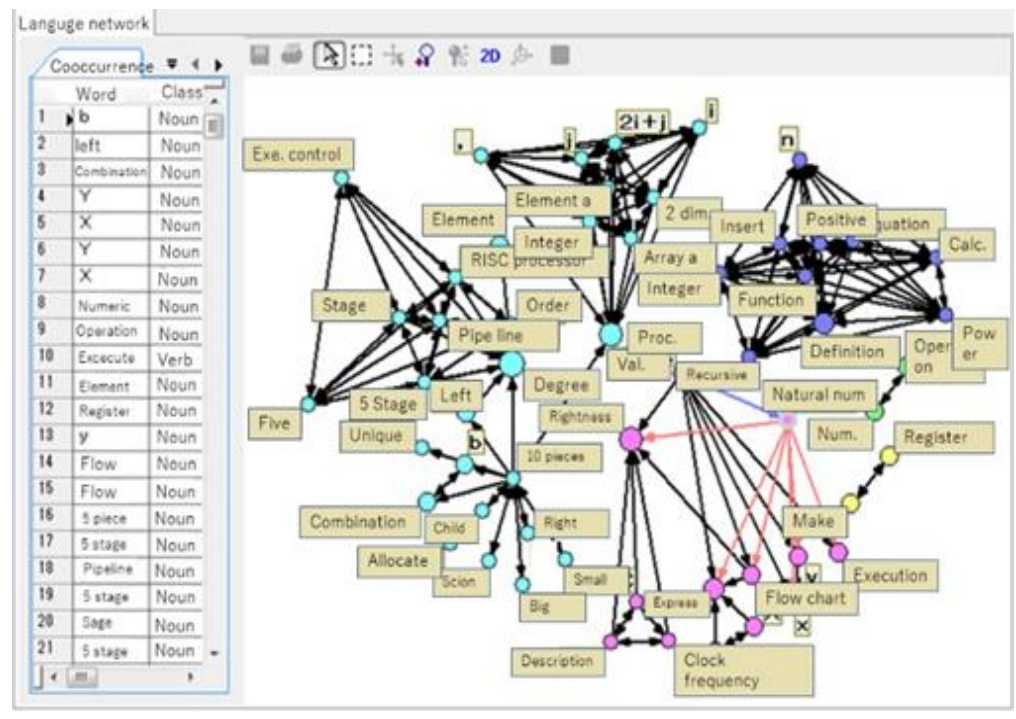

Figure 3 Example of co-occurrence words extraction and their graphic representation.

\subsection{Human Behavior Recognition and Control the Robot Actions}

Recently, many robots to perform the guidance and receptionists have been utilized in a variety of places [18,19], but those that move around are very few. In this research, we aim to develop a mobile system for the Pepper robot that can be easily introduced at educational sites, such as classrooms. Teachers are called by a show of hands in some cases of lectures. The Pepper robot needs ability to recognize the show of hands and move to the hand's position by following the black line. We developed a function to recognize the students who are raising their hands in the classroom using skeleton detection from monocular camera images. We were able to recognize the show of hands from the $\mathrm{Y}$ axis information of their shoulders, elbows, and wrists. In addition, we were able to determine which columns they were sitting from the XY information of their neck. In order to trace a line for Pepper, we developed a function to allow Pepper to discriminate black tape affixed to surfaces in a classroom in directions using deep learning. The deep learning network was made by using transfer learning the learned VGG16 network. Figure 4 shows moving robot by recognition of student's hand state. 


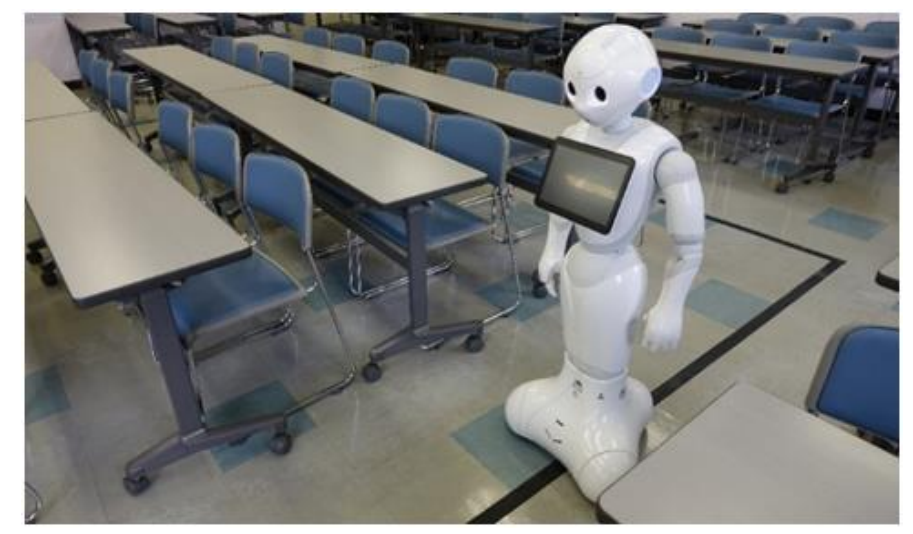

Figure 4 Moving robot by recognition of student's hand state.

\subsection{Human Education Based on Mental State}

In education, not only promoting effective understanding but also teaching behavior taking into consideration the learner's mental state becomes important. In order to conduct teaching behavior at the same time as analyzing the mental state, we need robots and agents. In this research, we analyze the influence of robot's own teaching behavior on learner's mental state. Furthermore, we propose an environment including a teacher robot, a TA robot and student robot (who is a partner robot).

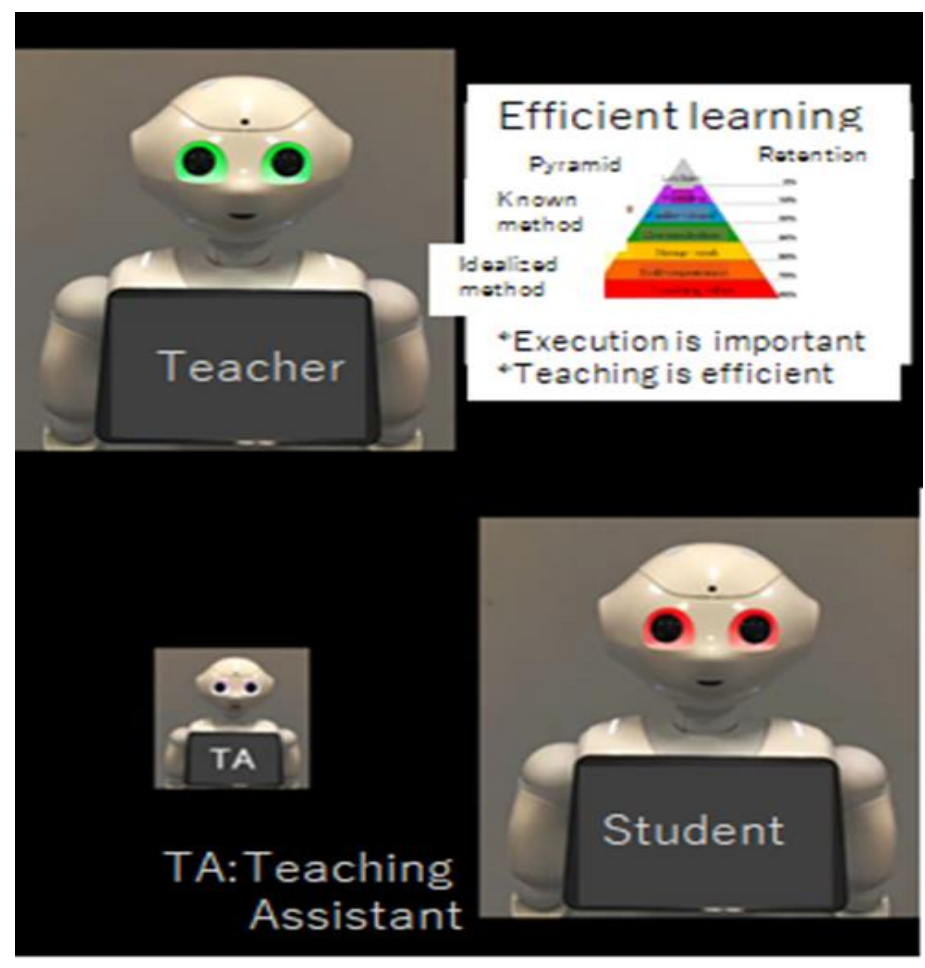

Figure 5 Color changing experiment during education.

\section{Results}

First, utterance mind expression for a communication system with a robot was applied in our system. We verify that investigate whether it is possible to facilitate communication with people by implementing "utterance mind" in emotion recognition through the humanoid Pepper.

The second element is estimation of the student condition using heartbeat. To detect the heartbeat, we applied a fuzzy spiking neural network as a pulse oscillator to generate a simulated heartbeat signal. Furthermore, we conducted an 
experiment in a real environment. In the experiment, the proposed system detected the change in the LF-HF ratio corresponding to the subject's psychological state.

The third element is data mining for discovering educational rules from log data. We executed apriori-based data mining to discover educational rules derived from experiments using robots proposed for educational communication. The results are promising in terms of functionality and time performance. In the next stage, we are planning to apply the algorithm to discovering rules from the log data obtained from actual experiments using education and communication robots.

The forth element is recognition of student's behaviours. We developed analysis function by student skeleton using OpenPose. Then we can recognize the behavior of raising hand. Teaching assistant robot is needed to move close position for rising hand student. We developed moving function to such student using line trace. We applied template matching of deep learning for line trace.

The fifth element is human education. In the first experiment, we investigated the influence of some statements ("This is important point." and "Be quiet.", etc.) with colored eyes in robots. As a result, it was revealed that the presented color affects the learner's impression, especially impression changes depending on learner's way of thinking about learning. In the second experiment, we investigated the impression when the robot speaks with colored eyes using script of an actual teacher. As a result, it was revealed that the presented color influences learner's mental state also in the context of the actual lesson. Figure 5 shows Color changing experiment during education.

\section{Conclusion}

We researched and developed the main element of human education and communication assist using robotics. Color phycology effects were observed by robot education. Our base technologies are human agent interaction, biological analysis, text mining, deep learning, robot control and education with mental state method. We will integrate these technologies and will build high levels human education assistance and communication robotics system based on color science phycology in the near future.

\section{Compliance with ethical standards}

\section{Acknowledgments}

This work was supported by "FY2016 MEXT Private University Research Branding Project" and one research of "Research and development of educational and communicational robot system" approved by the Council for Promotion of Universal Future Society project at MEXT, Japan.

\section{Disclosure of conflict of interest}

To the best of our knowledge, the named authors have no conflict of interest, financial or otherwise.

\section{References}

[1] Fukuda T and Iritani G. (1995). Construction mechanism of group behavior with cooperation. In IEEE/RSJ IROS, 535-542.

[2] Asama H, Ozaki K, Ishida Y, Yokita K, Matsumoto A, Kaetsu H and Endo I. (1994). Collaborative team organization using communication in a decentralized robotic system. In IEEE/RSJIROS, 816-823.

[3] Caloud P, Choi W, Latombe J-C, Pape Le C and Yin M. (1990). Indoor automation with many mobile robots. In IEEE/RSJ IROS, 67-72.

[4] Steels L. (1990). Cooperation between distributed agents through selforganization. In European Workshop on Modelling Autonomous Agents in a Multi-Agent World, 175-195.

[5] Kube CR and Zhang H. (1992). Collective robotic intelligence. In Proc. Simulation of Adaptive Behavior, 460-468.

[6] Dudek G, Jenkin M, Milios E and Wilkes D. (1993). A taxonomy for swarm robots. In IEEE/RSJ IROS, 441-447.

[7] Donald BR. (1995). On information invariants in robotics, Artificial Intelligence 72, 217-304, 
[8] Andrew G, Barto AG, Sutton RS and Watkins CJCH. (1989) Learning and Sequential Decision Making, LEARNING AND COMPUTATIONAL NEUROSCIENCE, MIT Press, 539-602.

[9] Littman M. (1994). Markov games as a framework for multi-agent reinforcement learning. In Proceedings of the International Machine Learning Conference, 157-163.

[10] Parker LE. (1994). Heterogeneous multi-robot cooperation, Ph.D. Dissertation, MIT EECS Dept.

[11] Cao YU, Fukunaga AS and Kahng AB. (1997). Cooperative Mobile Robotics: Antecedents and Directions, Autonomous Robots, 4, 7-27.

[12] Fujimoto K, Sasaki T, Sugaya M, Yoshimi T, Mizukawa M and Matsuhira N. (2016). A Collaborative Task Experiment by Multiple Robots in a Human Environment Using the Kukanchi System, 9th International Conference, ICIRA 2016, Part II, 276-282.

[13] Asada M. (2009). Cognitive Developmental Robotics: A Survey, IEEE Trans. Auto. Ment. Develop, 1(1), $12-34$.

[14] Ayash AA, Kane RT, Smith D and Armytage PG. (2015). The influence of color on student emotion, heart rate, and performance in learning environments, Color Research and Application, 41(2), 196-205.

[15] Kumar S, Sterkenburg J, Diekfuss J and Jeon M. (2013). Color Effects on Students' Emotionsand Task PerformanceinaWeb-based Learning Management System, in Proceedings of the International Conference on Multimedia and Human Computer InteractionToronto.

[16] Honya T, Katagami D, Yuasa M, Obo T, Ogai Y, Sone J, Tomoto T and Udagawa Y. (2018). Evaluation of robot's behavior to human based on understanding the situation using utterance mind, HUMAN COMMUNICATION GROUP SYMPOSIUM 2018 HCG2018-B-2-5.

[17] Kurihara Y and Watanabe K. (2012). Development of unconstrained heartbeat and respiration measurement system with pneumatic flow, IEEE Transactions on Biomedical Circuits and Systems, 6 (6), 596-604.

[18] Ishikawa R, Oishi T and Ikeuchi Katsushi. (2018). Offline and Onlinecalibration of Mobile Robot and SLAM Device for Navigation, eprint arXiv: 1804.04817.

[19] Suddrey G, Jacobson A and Ward B. (2018). Enabling a Pepper Robot to provide Automated and Interactive Tours of a Robotics Laboratory, eprint arXiv: 1804.03288.

\section{How to cite this article}

Sone J, Katagami D, Ogai Y, Tomoto T, Obo T and Udagawa Y. (2020). Feasiblity study of psychological approach using robotics for education and communication. Global Journal of Engineering and Technology Advances, 2(1), 08-13. 